\title{
Influence of Different Preparation Processes on the Mechanical Properties of Carbon Nanotube-Reinforced Copper Matrix Composites
}

\author{
C. H. Guo, Z. J. Zhan, ${ }^{1}$ and D. D. Zhang
}

State Key Laboratory of Metastable Materials Science \&Technology, Yanshan University, Qinhuangdao, Hebei, China

1 zjzhan@ysu.edu.cn

Experiments on the uniform distribution of carbon nanotubes in the copper matrix using different ball milling methods were performed. The effect of different preparation processes for carbon nanotube (CNT)-reinforced copper matrix composites on their conductivity and mechanical properties was also analyzed. High-performance carbon nanotube-reinforced copper matrix composites containing 1 vol.\% CNT by the best preparation process termed flake ball milling (FBM) possess good interface bonding strength not only with CNT evenly dispersed in the copper matrix but also with the CNT morphology and structure having the utmost integrity. Tensile strength increased by 30\% with an elongation of $26 \%$ and electrical conductivity of $85 \%$ IACS as compared to a pure copper block prepared from the same FBM powders.

Keywords: carbon nanotube-reinforced copper matrix $(\mathrm{CNT} / \mathrm{Cu})$ composites, flake ball milling, high-energy ball milling, ball milling, spark sintering technique.

Introduction. Since the discovery of CNTs by Lijima in 1991, carbon nanotubes have shown superb mechanical properties and electrical performance, and have proved to be good reinforcements for composites, both in theory and experiments [1-3]. However, there are still several problems to solve, the first problem being the uniform dispersion of CNTs. There are strong van der Waals forces between CNTs, therefore CNTs agglomerate quite easily and it is hard to ensure their uniform distribution in the metal matrix. There are several factors, which ensure the incompatibility between CNTs and copper ball particles: dimension incompatibility (from 1D to 3D), size incompatibility (from nano- to microscale), and surface property incompatibility (from hydrophilic to hydrophobic). The second problem is the interface bonding between $\mathrm{CNT}$ and $\mathrm{Cu}$. Carbons in CNTs are of $\mathrm{SP}^{2}$ hybrid structure with a low activity. They meet difficulty in forming effective interface bonding with metals, whose lattice structures are very different. The last problem is the structural integrity of CNTs. During the preparation process, CNT structures tend to be destroyed, which deteriorates their mechanical properties and strengthening effect.

Many preparation methods have been developed to fabricate CNT-reinforced metal matrix composites, including molecular level mixing (MLM) [4, 5], nanoscale dispersion [6], in situ approach [7], flake powder metallurgy [8-10], high-energy ball milling (HEBM) [11], high-pressure torsion and friction stir processing [12], and rolling [13]. Nickel-coated CNTs have been mixed with copper powder to be prepared by cool pressed-vacuum sintering-rolling-vacuum annealing [5]. Moreover, composite materials exhibit a good wear resistance and low friction performance. $\mathrm{CNT} / \mathrm{Cu}$ composites have been synthesized using HEBM and high-ratio differential speed rolling (HRDSR) [14]. Although such composites demonstrate the improved mechanical properties, the morphology and structure of CNTs are damaged. The strengthening efficiency of CNTs and the elongation of composites are unsatisfactory.

A unique method called MLM has been proposed for producing CNT-metal composites, such as $\mathrm{CNT} / \mathrm{Cu}$ [7], CNT/Al-Cu [15, 16], and CNT/Ni [17]. This processing procedure involves mixing CNTs into the copper-salt bath, and deposited $\mathrm{Cu}$ on their 
surface, followed by drying and calcination in air. The obtained stable CNT/CuO powders are reduced in hydrogen atmosphere, and then sintered by spark sintering technique (SPS). $\mathrm{CNT} / \mathrm{Cu}$ composites are strengthened by load transfer and grain refinement of the $\mathrm{Cu}$ matrix [18]. Load-transfer efficiency is excellent due to chemically bonded oxygen atoms at the CNT/Cu interface [19]. However, this method involves relatively complicated processing steps, such as reduction in hydrogen.

A new method called flake powder metallurgy (PM) is used to produce $\mathrm{CNT} / \mathrm{Al}$ composites [12-14]. It allows one to achieve a uniform distribution of CNTs at the flake Al powder surface by slurry blending. By their surface modification with a polyvinyl alcohol hydrosol (PVA), flake PM has achieved high compatibilities of Al powders with CNTs, in terms of both surface properties and geometries. Such method can be refined to manufacture similar composites with a variety of nano-reinforcements in shapes and different matrices.

In this paper, a new dispersion method is used to obtain a high interface bonding between CNTs and $\mathrm{Cu}$ matrix, as well as to provide the effective reduction of the aggregation of CNTs, and to improve the compatibility of CNTs. The technique is simple, efficient, and suitable for industrial mass production.

1. Experimental Procedure. The surface of multi-walled CNTs of approximately $50 \mathrm{~nm}$ in diameter and of $10-20 \mu \mathrm{m}$ in length is designed with hydroxyl $(-\mathrm{OH})$ and carboxyl $(-\mathrm{COOH})$ groups and is supplied by Chengdu Organic Chemistry Co. Ltd., China. The density of CNTs quoted by the manufacturer is $2.1 \mathrm{~g} / \mathrm{cm}^{3}$. Electrolyzed dendritic $\mathrm{Cu}$ powders of $45 \mu \mathrm{m}$ in size are used. CNTs/Cu composites are made in four steps: making flake $\mathrm{Cu}$ powders, coating of CNTs with $\mathrm{Cu}$, mixing flake $\mathrm{Cu}$ powders with coated CNTs by ball milling (BM), and eventual spark plasma sintering.

1.1. Electroless Copper-Plating of CNTs. In order to reduce the aggregation of CNTs and improve the interface bonding and compatibility of CNTs, CNTs are coated with $\mathrm{Cu}$. To transfer the catalytic sites to the surface of CNTs for $\mathrm{Cu}$ deposition, CNTs are pretreated using sensitization and activation [9]. CNTs are dispersed with 1 wt.\% sodium dodecyl benzene sulfonate as surfactant for ultrasonic processing $(10 \mathrm{~min})$, and then are added to an aqueous solution containing $8.9 \cdot 10^{-3} \mathrm{M} \mathrm{SnCl}_{2}$ and $2.4 \cdot 10^{-3} \mathrm{M} \mathrm{HCl}$ to be sensitized for $10 \mathrm{~min}$ and, finally, to the aqueous solution containing $1.2 \cdot 10^{-3} \mathrm{M} \mathrm{PdCl}_{2}$ and $1.2 \cdot 10^{-2} \mathrm{M}$ $\mathrm{HCl}$ to be activated for $30 \mathrm{~min}$. Sensitization makes it possible to absorb $\mathrm{Sn}^{2+}$ colloid layer by CNTs surface, where $\mathrm{Sn}^{2+}$ provides reduction restoration of $\mathrm{Pd}^{2+}$ into nanoscale $\mathrm{Pd}$ particles in the activation process. The catalytic performance of $\mathrm{Pd}$ particles facilitates deposition of copper.

Numerous experiments have proved the the best coating quality was obtained, when the appropriate plating temperature was $60^{\circ} \mathrm{C}$, $\mathrm{pH}$ value was 11 and the plating proportion of the plating bath and CNTs was 2L:1 g. After plating, the CNT surfaces have been almost evenly coated with copper of about $50 \mu \mathrm{m}$ in thickness. CNTs' wettability with matrix and interface bonding is also drastically improved.

1.2. BM Technique. Ball milling is a technique for the dispersion of CNTs in the matrix, which influences the structural integrity of CNTs. Several ball milling processes are adopted as follows:

1) Flake ball milling: $\mathrm{Cu}$ powders have been prepared by ball milling at a speed of $200 \mathrm{rpm}$ with ball-to-powder weight ratio (BPR) 8:1 for $8 \mathrm{~h}$ to obtain flake powders. The coated CNTs and the flake $\mathrm{Cu}$ powders with BPR 8.5:1 have been prepared by ball milling at a speed of $150 \mathrm{rpm}$ for $2 \mathrm{~h}$. This process is referred to as FBM.

2) High-energy ball milling: The coated CNTs and $\mathrm{Cu}$ powders have been planetary ball milled at a speed of $200 \mathrm{rpm}$ with BPR 8.5:1 for $10 \mathrm{~h}$. This process is referred to as HEBM.

3) Ball milling: The coated CNTs and $\mathrm{Cu}$ powders have been planetary ball milled at a speed of $150 \mathrm{rpm}$ with BPR 2:1 for $4 \mathrm{~h}$. This process is called BM. 
1.3. Spark Plasma Sintering. During sintering the material undergo densification. Sintering temperature, pressure, holding time, cooling rate and other factors control the microstructure and properties of materials. Numerous tests have been performed to find the optimal SPS process as follows: preloading stress $20 \mathrm{MPa}$, sintering temperature $700^{\circ} \mathrm{C}$, heating rate $40 \mathrm{~K} / \mathrm{min}$, sintering pressure $40 \mathrm{MPa}$, and heat preservation for $5 \mathrm{~min}$. Using the above-mentioned sintering process, four sets of specimens are prepared as follows:

(i) the FBM composite block has been prepared by the FBM CNT/Cu powders and the above SPS process; (ii) the HEBM composite block has been prepared by the HEBM $\mathrm{CNT} / \mathrm{Cu}$ powders and the same SPS process; (iii) the BM composite block has been prepared by the BM CNT/Cu powders and the same SPS process; (iv) the BM $\mathrm{Cu}$ block has been prepared by the BM $\mathrm{Cu}$ powders and the same SPS process.

2. Results and Discussion. The microstructure of $\mathrm{CNT} / \mathrm{Cu}$ composite has been examined using field emission scanning microscope (FESEM) (Hitachi S-4800). Highresolution transmission electron microscopy (HRTEM) has been carried out using in a FEI TECNAI G2 F30 microscope operated at $300 \mathrm{kV}$ with an energy dispersive X-ray microanalyzer (EDAX). The structural integrity of CNTs is analyzed using Raman spectroscopy (Renishaw, inVia) with $543 \mathrm{~nm}$ solid-state laser as an excitation source. Phase analysis of the coated CNTs has been conducted by X-ray diffraction (XRD) (Ricoh, $\mathrm{D} / \max -2500)$ with $\mathrm{CuK} \alpha$ radiation. Using the four-probe method, the electrical conductivity of the composites was measured. Tensile strength tests have been performed using TH5000 with an initial strain rate of $1 \cdot 10^{-3} \mathrm{~s}^{-1}$ at room temperature. Sub-size dog-bone shape specimens have been machined along the cross-sectional direction of the compacts with the gauge length of $12 \mathrm{~mm}$ and width of $2 \mathrm{~mm}$.

2.1. Analysis of Chemical Plating Effect. Figure 1a shows the FESEM micrograph of the original CNTs of about $50 \mathrm{~nm}$ in diameter. The tangled networks of CNTs can hardly be separated only by wet mixing in some surfactant solutions. Therefore a single wet mixing CNTs with $\mathrm{Cu}$ powders was infeasible, and surface modifying of CNTs had to be used as well. Figure $1 \mathrm{~b}$ demonstrates the morphology of coated CNTs. The nano-sized $\mathrm{Cu}$ particles are closely attached along the tangled CNTs and form the coarse $\mathrm{Cu}$ layers on the nanotube surfaces. The size of the deposited $\mathrm{Cu}$ particles is calculated to be between 50 and $100 \mathrm{~nm}$. From the XRD results of the coated CNTs (Fig. 1c), it can be seen that there is a small amount of $\mathrm{Cu}_{2} \mathrm{O}$ as by-products of the chemical plating reaction.

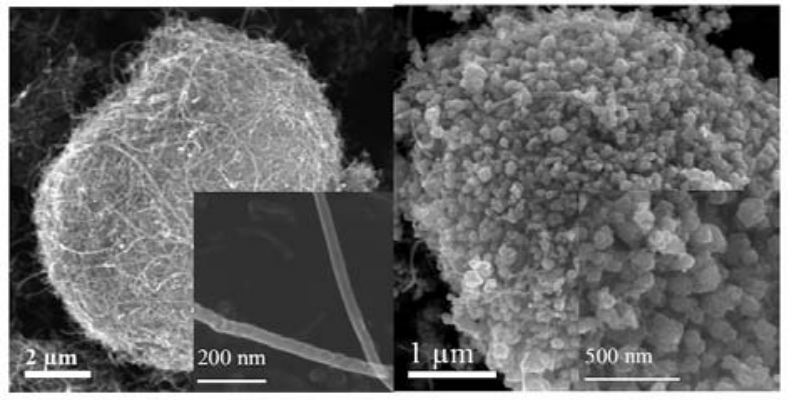

a

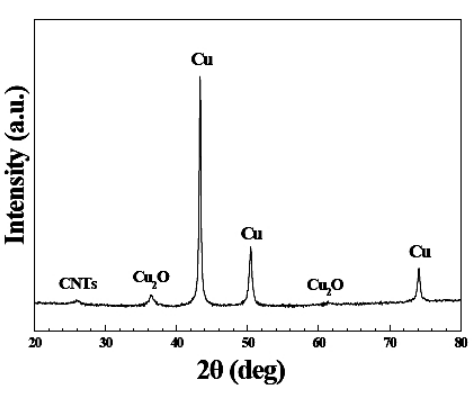

C

Fig. 1. FESEM and XRD of CNTs: (a) original CNTs; (b) coated CNTs; (c) XRD of coated CNTs.

2.2. Analysis of Ball Milling Effect. Figures 2a-c shows the images of the BM powders. As it is shown in Fig. 2a, the BM Cu powders kept their branch structures. As it is shown in Fig. 2b, c, CNTs were bended and attached to the $\mathrm{Cu}$ particle surfaces, rather than embedded into the $\mathrm{Cu}$ matrix. Moreover, in some areas, agglomerate CNTs are observed. Due to geometric incompatibility between $\mathrm{Cu}$ powders and $\mathrm{CNTs}$, the branch $\mathrm{Cu}$ powders 


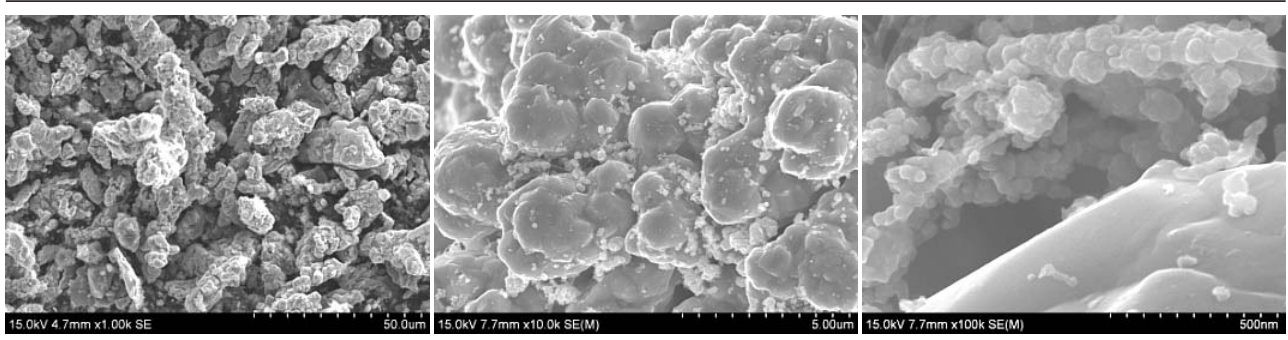

a

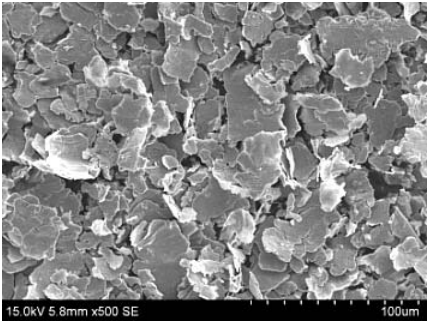

d b

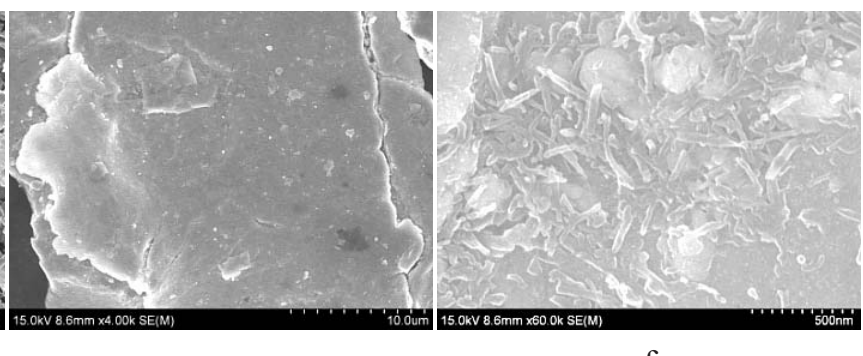

e

Fig. 2. Morphology of the CNT/Cu powders containing 1vol\% CNTs: (a) BM powders; (b), (c) high-magnification FESEM for BM powders; (d) FBM powders; (e), (f) high-magnification FESEM for FBM powders.

have large curvatures and small surface area. Meanwhile, the CNTs are one-dimensional nanofibers with a large surface area.

Figures $2 \mathrm{~d}-\mathrm{f}$ show the morphology of $\mathrm{FBM} \mathrm{CNT} / \mathrm{Cu}$ powders. It can be seen that CNTs are uniformly distributed on the surface of the flake $\mathrm{Cu}$ powders or embedded closely into the $\mathrm{Cu}$ particle surfaces, while the CNTs are cut into shorter ones, which contribute to the uniform dispersion of CNTs. As the ball milling energy of FBM is relatively low, most of the deposited $\mathrm{Cu}$ nanoparticles have not peeled-off. Due to the flake shape and increased area of the flake $\mathrm{Cu}$ powders, the adsorption capacity of FBM CNTs is much better than those of BM. The FBM Cu powders overcame the curvature effect of the dendrite shape and improve the geometric compatibility of $\mathrm{Cu}$ powders with CNTs $[19,20]$. FBM-made CNTs and copper powders manifest good compatibilities in both geometry and surface adsorption.

2.3. Analysis of the Stress-Strain Curve and Raman Spectroscopy. Figure 3 shows the true tensile stress versus strain curves of the FBM CNT/Cu composite containing 1 vol.\% CNTs, BM CNT/Cu composite containing 1 vol.\% CNTs, and the CNTs-free FBM Cu. The mechanical properties are presented in Table 1. Curves in Fig. 3 manifest a sharp turn at 0.2 (true strain) points, which separate the elastic and plastic deformation stages. The tensile strength of the FBM CNT/Cu composite containing 1 vol.\% CNTs was $269 \mathrm{MPa}$ increases by almost $30 \%$, as compared with that of FBM Cu. The tensile strength of the BM $\mathrm{CNT} / \mathrm{Cu}$ composite containing 1 vol.\% CNTs is $235 \mathrm{MPa}$, which is larger than that of pure $\mathrm{Cu}$, but much less than that of the FBM one. This indicates that thw FBM method is better than the BM method aimed at efficient dispersion of CNTs. The FBM composites demonstrate an elongation of $26 \%$.

It is seen from Table 1 that the electrical conductivity of $\mathrm{Cu}$ is $98 \%$ IACS $\%$ and that of $\mathrm{FBM} \mathrm{CNT/Cu}$ composite containing 1 vol.\% CNTs is $85 \%$, while for $\mathrm{BM} \mathrm{CNT/Cu}$ composite containing 1 vol. $\%$ CNTs it is $91 \%$. Thus, the electrical conductivity of the FBM composites is lower than that of the BM composites. This is due to CNTs at the $\mathrm{Cu}$ grain boundaries, which will scatter the charge carrier. The FBM method provides a uniform 
$\mathrm{T}$ a b 1 e 1

Some Properties of the Specimens

\begin{tabular}{|c|c|c|c|c|c|}
\hline \multirow[t]{2}{*}{ Sample } & \multirow{2}{*}{$\begin{array}{l}\text { Tensile } \\
\text { strength } \\
(\mathrm{MPa})\end{array}$} & \multirow{2}{*}{$\begin{array}{c}\text { Electrical } \\
\text { conductivity } \\
\text { (IACS\%) }\end{array}$} & \multirow{2}{*}{$\begin{array}{l}\text { Strengthening } \\
\text { efficiency } \\
\text { (R) }\end{array}$} & \multicolumn{2}{|c|}{ Raman ID:IG } \\
\hline & & & & Powder & Compact \\
\hline $\mathrm{FBM} \mathrm{Cu}$ & 208 & 98 & - & - & - \\
\hline BM 1 vol.\% CNT/Cu & 235 & 91 & 18 & 0.805 & 0.826 \\
\hline FBM 1 vol. $\% \mathrm{CNT} / \mathrm{Cu}$ & 269 & 85 & 29 & 1.040 & 1.188 \\
\hline
\end{tabular}

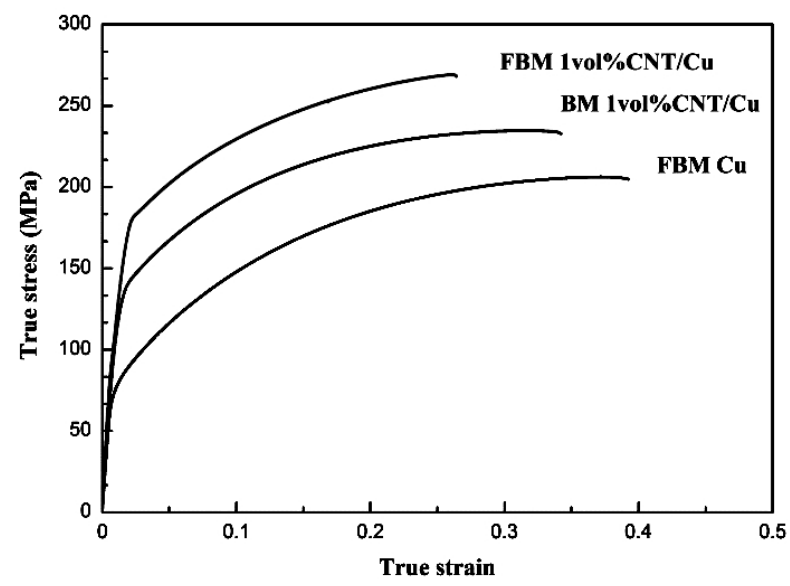

Fig. 3. True stress-strain curves of the FBM pure $\mathrm{Cu}$, BM CNT/Cu composite containing 1 vol.\% CNTs, and FBM CNT/Cu composite containing 1 vol.\% CNTs.

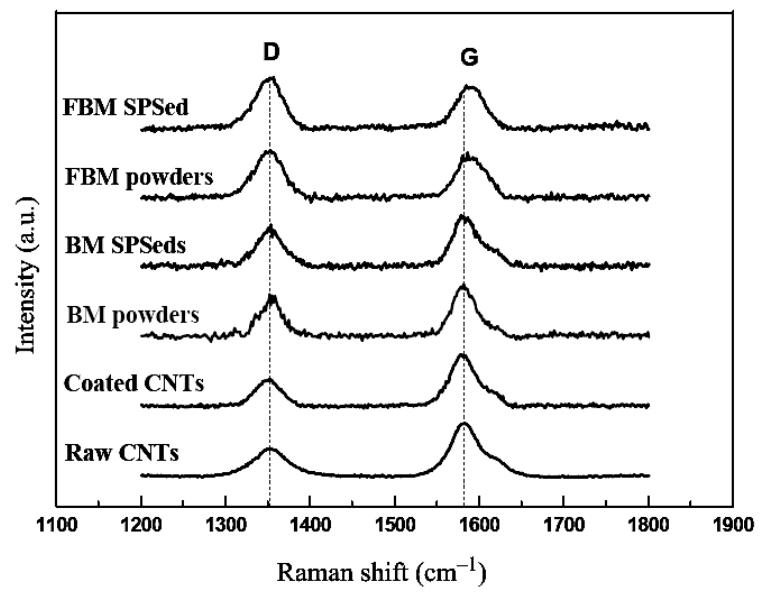

Fig. 4. The Raman spectra of the CNTs, the coated CNTs, the BM CNTs/Cu powders containing 1 vol.\% CNTs, and FBM CNTs/Cu powders containing 1 vol.\% CNTs.

dispersion of the CNTs, increases the grain boundary electron scattering, and reduces the electrical conductivity.

Figure 4 shows the Raman spectra of the original CNTs, coated CNTs, the BM $\mathrm{CNT} / \mathrm{Cu}$ powders containing 1 vol.\% CNTs, and $\mathrm{FBM} \mathrm{CNT/Cu}$ powders containing 1 vol.\% CNTs. The Raman peaks around $1578 \mathrm{~cm}^{-1}$ denote the G-brand (graphite), while $1352 \mathrm{~cm}^{-1}$ represents the D-band (defect) [20]. As seen from Fig. 4, the Raman peak relative intensity 
ratios of the D-band and G-band (ID:IG) are calculated and demonstrated in Table 1. Those results provide the information on the quality of the CNTs.

The ID:IG ratio of the original CNTs is 0.530 , while the respective value of the coated CNTs is 0.543 . It means that the electroless deposition process allowed one to preserve the maximal structural integrity of CNTs.

Furthermore, the peaks and the ID:IG values of the composite powders made by FBM or BM methods are similar to those of their SPS blocks. This indicates that the destruction to the CNT structure is caused mainly by the ball milling rather than by sintering. Due to high ball milling energy, FBM CNTs are damaged more seriously than BM ones. The G-band peak of the FBM powders is shifted to $1584 \mathrm{~cm}^{-1}$. The value of ID:IG for the FBM sintered composites (1.118) is higher than those prepared by a nanoscale dispersion (0.858) [6] and slurry bending (0.72) [20].

The aspect ratio of the original CNTs in this study is in the range of 200-400, which is much higher than that for slurry bending (approximately 50) [19, 20]. The tangled networks of the original CNTs must be separated by ball milling. In addition, the ID:IG value of FBM CNTs is better than those of the HEBM CNT/Cu composites containing 3 vol.\% CNTs (1.24) combined with HRDSR. In order to maximize the strengthening efficiency of CNTs in the metal matrix, it is important to find a suitable method to keep the balance between the uniform dispersion and quality of CNTs.

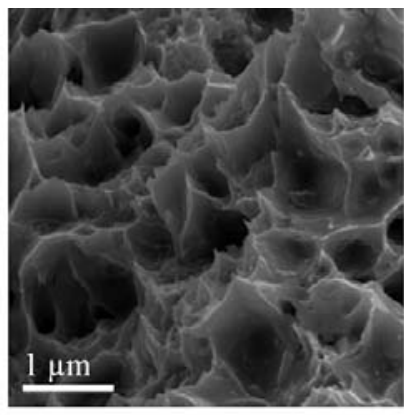

a

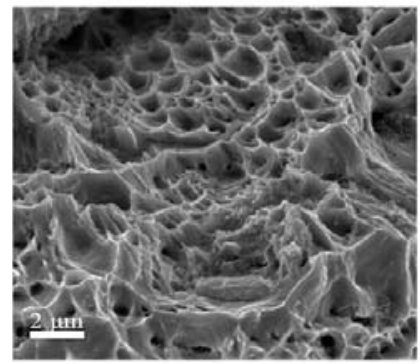

d

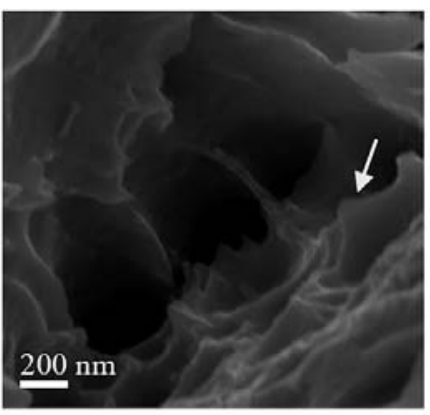

b

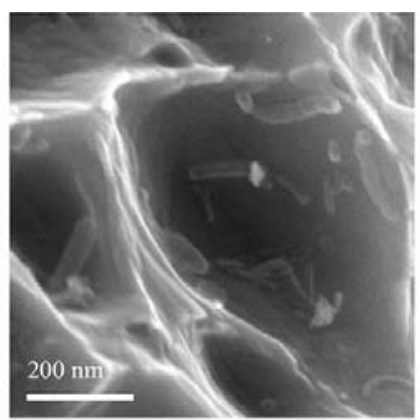

e

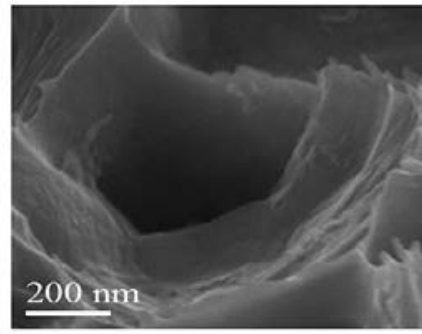

c

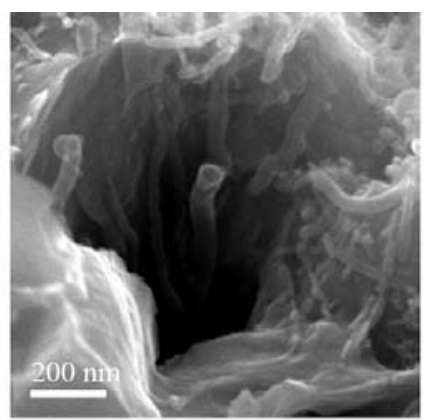

$\mathrm{f}$

Fig. 5. FESEM micrographs of the CNT/Cu composite fracture surface after tensile testing: (a-c) FESEM micrographs of FBM CNT/Cu composite; (d-f) FESEM micrographs of BM CNT/Cu composite.

2.4. Analysis of the FESEM Micrographs after Tensile Testing. Figure 5 illustrates the FESEM micrographs of FBM CNT/Cu composite and the BM ones after tensile testing. As it is shown in Fig. 5e, CNT bridging is formed in the matrix, which plays an important role in the interfacial load transfer from $\mathrm{Cu}$ matrix to $\mathrm{CNTs}$. There are numerous dimples caused by ductile fracture in the fracture surface (Fig. 5d). A detailed observation shows that CNTs appeared in the dimple walls (as shown in Fig. 5e, f, which helps one to increase the 
tensile strength of composites by improving the load transfer from matrix to CNTs. The CNTs in FBM composites are found to be well-dispersed in the matrix (Fig. 5d-f). However, there are some CNTs agglomerations at BM composite fracture surfaces in Fig. $5 \mathrm{a}-\mathrm{c}$. CNTs agglomerations in the dimples act as pores in the matrix and as nucleation sites for cracks, which eventually lead to failure under tensile load. Due to poor dispersion of CNTs in the BM composites, the load transfer efficiency of CNTs is reduced.

Finally, homogeneously dispersed CNTs in FBM CNT/Cu have a strong interfacial bonding with $\mathrm{Cu}$ matrix, which makes it possible to improve the mechanical properties of the composites.

Conclusions. The aim of this study is to produce $\mathrm{CNT} / \mathrm{Cu}$ composites with a homogeneous distribution of CNTs by using the FBM method and SPS. High-energy ball milling is used to rapidly obtain flake $\mathrm{Cu}$ powders and the coated CNTs. Moreover, high compatibility of $\mathrm{Cu}$ with $\mathrm{CNTs}$ powders was achieved, which results in the improved load-transfer efficiency of CNTs in the $\mathrm{Cu}$ matrix. A small-scale addition of 1 vol.\% CNTs contributed to $30 \%$ increase in the tensile strength and caused $85 \%$ IACS of the electrical conductivity. A high strengthening efficiency of CNTs is attributed to the interfacial oxygen atoms that are chemically bonded between the CNTs and $\mathrm{Cu}$, which can be controlled in the electroless $\mathrm{Cu}$ deposition process. As a result, FBM is found to be a simple and lucrative method to produce CNTs-reinforced $\mathrm{Cu}$ matrix composite with a high aspect ratio.

Acknowledgments. The authors would like to thank Z. C. Wang, Y. T. Wen and other members of the School of National Defense Science \& Technology, Yanshan University. This study was supported by the National Basic Research Program of China (2010CB71600).

1. Y. S. Chen, A. Malkovskiy, X. Q. Wang, et al., "Selection of single-walled carbon nanotube with narrow diameter distribution by using a PPE-PPV copolymer," ACS Macro Lett., 1, 246-251 (2012).

2. J. D. Leeds, J. T. Fourkas, and Y. Wang, "Achieving ultrahigh concentrations of fluorescent single-walled carbon nanotubes using small-molecule viscosity modifiers," Small, 9, 241-247 (2013).

3. X. Q. Wang and Y. Pang, "Selective dispersion of single-walled carbon nanotubes by a cationic surfactant," RSC Adv., 3, Issue 47, 25097-25102 (2013).

4. S. Rotkin and Y. Gogotsi, "Analysis of non-planar graphitic structures: from arched edge planes of graphite crystals to nanotubes," Mater. Res. Innov., 5, 191-200 (2002).

5. S. I. Cha, K. T. Kim, S. N. Arshad, et al., "Extraordinary strengthening effect of carbon nanotubes in metal-matrix nanocomposites processed by molecular-level mixing," Adv. Mater., 17, 1377-1381 (2005).

6. H. S. Kwon, M. Estili, K. Takagi, et al., "Combination of hot extrusion and spark plasma sintering for producing carbon nanotube reinforced aluminum matrix composites," Carbon, 47, 570-577 (2009).

7. C. He, N. Q. Zhao, C. S. Shi, et al., "An approach to obtaining homogeneously dispersed carbon nanotubes in $\mathrm{Al}$ powders for preparing reinforced Al-matrix composites," Adv. Mater., 19, 1128-1132 (2007).

8. X. Z. Kai, Z. Q. Li, G. L. Fan, et al., "Strong and ductile particulate reinforced ultrafine-grained metallic composites fabricated by flake powder metallurgy," Scripta Mater., 68, 555-558 (2013).

9. L. Jiang, Z. Q. Li, X. Z. Kai, et al., "An approach to the uniform dispersion of a high volume fraction of carbon nanotubes in aluminum powder," Carbon, 49, 1965-1971 (2011). 
10. L. Jiang, Z. Q. Li, G. L. Fan, et al., "The use of flake powder metallurgy to produce carbon nanotube (CNT)/aluminum composites with a homogenous CNT distribution," Carbon, 50, 1993-1998 (2012).

11. H. J. Choi, G. B. Kwon, G. Y. Lee, and D. H. Bada, "Reinforcement with carbon nanotubes in aluminum matrix composites," Scripta Mater., 59, 360-363 (2008).

12. H. Li, A. Misra, Z. Horita, et al., "Strong and ductile nanostructured Cu-carbon nanotube composite," Appl. Phys. Lett., 95, 071907-1-071907-3 (2009).

13. Z. Y. Liu, B. L. Xiao, W. G. Wang, and Z. Y. Ma, "Developing high-performance aluminum matrix composites with directionally aligned carbon nanotubes by combining friction stir processing and subsequent rolling," Carbon, 62, 35-42 (2013).

14. S. J. Yoo, S. H. Han, and W. J. Kim, "A combination of ball milling and high-ratio differential speed rolling for synthesizing carbon nanotube/copper composites," Carbon, 61, 487-500 (2013).

15. D. H. Nam, Y. K. Kim, S. I. Cha, and S. H. Hong, "Effect of CNTs on precipitation hardening behavior of CNT/Al-Cu composites," Carbon, 50, 4809-4814 (2012).

16. D. H. Nam, S. I. Cha, B. K. Lim, et al., "Synergistic strengthening by load transfer mechanism and grain refinement of CNT/Al-Cu composites," Carbon, 50, 2417-2423 (2012).

17. J. Y. Hwang, B. K. Lim, J. Tiley, et al., "Interface analysis of ultra-high strength carbon nanotube/nickel composites processed by molecular level mixing," Carbon, 57, 282-287 (2013).

18. K. T. Kim, J. Eckert, S. B. Menzel, et al., "Grain refinement assisted strengthening of carbon nanotube reinforced copper matrix nanocomposites," Appl. Phys. Lett., 92, 121901-1-121901-3 (2008).

19. K. T. Kim, S. I. Cha, T. Gemming, et al., "The role of interfacial oxygen atoms in the enhanced mechanical properties of carbon-nanotube reinforced metal matrix nanocomposites," Small, 4, 1936-1940 (2008).

20. F. Wang, S. Arai, and M. Endo, "The preparation of multi-walled carbon nanotubes with a Ni-P coating by an electroless deposition process," Carbon, 43, 1716-1721 (2005). 